\title{
LA SOCIETÀ COOPERATIVA BRASILIANA
}

\section{THE BRAZILIAN COOPERATIVE PARTNERSHIP}

Danilo Borges dos Santos Gomes de Araujo

\author{
Dottore di ricerca in "Sistema giuridico romanistico e \\ unificazione del diritto" - "Università degli studi di Roma II \\ "Tor Vergata"”. Professore - "Escola de Direito de São Paulo" \\ della "Fundação Getulio Vargas" (Direito GV), Brasile. \\ danilo_de_araujo@hotmail.com
}

Ricevuto: 2015-11-02. Accolto: 2016-07-04.

Sommario: Considerando la vastità del tema e dei problemi che ne derivano, trattasi di esposizione relativamente sintetica sulla società cooperativa brasiliana, concentrandosi in particolar modo sui seguenti punti: (i) sviluppo storico-legislativo sul cooperativismo e sulla società cooperativa in Brasile; (ii) caratteri principali dello schema giuridicoorganizzativo della società cooperativa brasiliana; e (iii) numeri e dati statistici ed economici complessivi sulla attività economica svolta tramite le cooperative in Brasile e sulla diffusione (costituzione), in Brasile, di quel tipo societario chiamato "sociedade cooperativa". Il testo quindi si concentra esclusivamente sulla società cooperativa brasiliana, assente qualsiasi tentativo di confronto diretto con la disciplina della società cooperativa italiana, anche se gli esperti in materia possono indirettamente cogliere i contrasti tra l'uno e l'altro ordinamento giuridico, trovando somiglianze e differenze tra i rispettivi schemi legali della cooperativa.

Parole-chiave: Cooperativismo in Brasile - Società cooperativa brasiliana - Sviluppo storico-legislativo e caratteri dello schema societario - Dati statistici

Abstract: Considering the vastness of the topic and the related problems that may arise, the text is a relatively synthetic presentation on the Brazilian cooperativepartnership, focusing in particular on the following points: (i) historical and legislative development of the Brazilian cooperativism and the Brazilian cooperative partnership; (ii) the main traits of the Brazilian cooperative partnership legal and organizational framework; and (iii) overall statistical and economical numbers and data on the economic activity carried out by cooperatives in Brazil and on the spread, in Brazil, of the partnership type called "sociedadecooperativa". The text therefore 
focuses exclusively on the Brazilian cooperative partnership, absent any attempt to make a direct comparison with the Italian discipline, although experts in the field can indirectly capture the contrasts between one and the other legal systems, finding similarities and differences between their respective cooperative legal frameworks.

Keywords: Cooperativism in Brazil - Brazilian cooperative partnership Historical and legislative development and legal framewrok - Statistical data

\section{SVILUPPI STORICI-LEGISLATIVI DELLA SOCIETÀ COOPERATIVA IN BRASILE}

In Brasile, le prime cooperative emersero nel XIX secolo, soprattutto come cooperative di consumo. La storiografia evidenzia quanto segue: la "Cooperativa de Produção Teresa Cristina" (1847), in Paraná; la "Cooperativa de Consumo dosEmpregados da Companhia Paulista" (1887), in Campinas, São Paulo; la "Cooperativa de Consumo dosFuncionáriosdosFuncionários da Prefeitura de OuroPreto" (1889), in MinasGerais; e la "Cooperativa Militar de Consumo do Rio de Janeiro" $(1894)^{1}$. Nel 1902, ebbe origine la prima cooperativa di credito del paese (ed anche della America Latina), la "Caixa de CréditoRural de Petrópolis", in Rio Grande do Sul, in attività ancora oggi². Uno dei fattori che hanno contribuito alla realizzazione, lo sviluppo e il consolidamento del movimento cooperativo in Brasile è stato in grande afflusso de immigrati europei, in particolare italiani e tedeschi, alla fine del XIX secolo e l'inizio del XX secolo ${ }^{3}$, avendo gli immigrati portato il loro bagaglio culturale il lavoro associativo e l'esperienza delle attività famigliari comunitari, che li hanno motivati a organizzarsi in cooperative.

Dal punto di vista della legislazione, il primo riferimento alle cooperative che conosciamo è contenuto nel decreto $\mathrm{n}^{\circ} 796$, del 2 ottobre 1890, che trattò specificamente di una cooperativa organizzata dai militari, la "Sociedade Cooperativa Militar do Brasil". Solo all'inizio del XX apparvero, nel diritto brasiliano, norme di carattere generale sulle cooperative, la prima corrispondente al decreto $\mathrm{n}^{\mathbf{0}}$ 979,

1 Cf. J. Batista Brito Pereira, Da Sociedade Cooperativa, in D. Franciulli Netto, G. Ferreira Mendes \& I. Gandra da Silva Martins Filho (coordenadores), O Novo CódigoCivil. Homenagemao Professor Miguel Reale, 2. ${ }^{\text {a }}$ ed., São Paulo, 2006, p. 937. [= in Revista do TribunalSuperior do Trabalho, vol. 69, n. ${ }^{\circ}$ 2, Brasília, julho-dezembro de 2003, pp. 32-54.]

2 Cf. M.A. HenriquesPinheiro, Cooperativas de Crédito. História da Evolução Normativa no

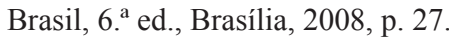

3 Solo per curiosità, si registra, in riferimento ai numeri di immigrazione italiana in Brasile, un'impressionante contingente di più di 1,4 milioni di immigrati trai 11884 e il 1933 (cf., in tal senso, le statistiche del documento del Ministério do Planejamento, Orçamento e Gestão, Instituto Brasileiro de Geografia e Estatística, Centro de Documentação e Disseminação de Informações, Brasil. 500 Anos de Povoamento, Rio de Janeiro, 2007, p. 226). 
del 6 gennaio 1903, la quale, nel suo art. 10, ha agevolato i sindacati rurali con la possibilità di organizzare banche agricole di credito, così come cooperative di produzione o di consumo, senzaperò prevedere maggiori dettagli sul tema ${ }^{4}$.

Successivamente, nacque quello che sarebbe probabilmente primo statuto brasiliano dedicato alle cooperative: il decreto $n^{\circ} 1637$, del 5 gennaio 1907, destinato a regolamentare il settore ancora nascente ${ }^{5}$. Questo decreto già delineò alcune caratteristiche delle cooperative, per stabilire: la variabilità del capitale; la non limitazione del numero di soci; l'inalienabilità delle azioni, quote o parti a terzi esterni all'azienda; e la non obbligatorietà di capitale per alcuni tipi di cooperative. Tuttavia, in questa occasioneancora non fu attribuita una forma propria alle società cooperative, che dovrebbero essere organizzate come società anonima, in nome collettivo o in accomandita.

Si aggiunga che questo passo legislativo dell'innesto della società cooperativa in Brasile è stato caratterizzato da una grande libertà operativa di quest'ultima, che non è subordinata ad alcuna agenzia statale ${ }^{6}$.

Nei decenni successivi, il settore ha conosciuto un'enorme espansione, attraverso la nascita di regole molto specifiche per alcuni tipi di cooperative, come le cooperative di credito di tipo Reiffeisen e di tipo Luzzatti, previste nel decreto ${ }^{\circ} 17339$, del 2 giugno 1926.

Il quadro legislativo seguente, risultato della maturazione dell'organizzazione cooperativa nel Paese, è stato il decreto $\mathrm{n}^{\circ} 22.239$, del 19 dicembre 1932, che ha modificato la legge del 1907 e ha definito l'inizio del periodo di consolidamento della idea de cooperativa in Brasile. ${ }^{7}$ Nonostante i difetti tecnici e terminologici, questo decreto ha stabilito i principi dottrinali delle cooperative e conservato, di regola, la loro libertà di stabilimento e di attuazione, favorendo una loro maggiore diffusione. ${ }^{8} \mathrm{Si}$ cercò di dare una forma propria alla società cooperativa, come si vede nella definizione giuridica del art. $2^{\circ}$ della riferita norma: "Associedadescooperativas, qualquerqueseja a sua natureza, civiloumercantil, sãosociedades de pessoas e não de capitais, de forma jurídica sui generis, que se distinguemdasdemaissociedades."

Tuttavia, non avendo previsto norme specifiche per la responsabilità dei soci e terzi, non avendo stabilito chiaramente la sua

4 Cf. J. Batista Brito Pereira, Da Sociedade Cooperativa cit., p. 941; e . M.A. HenriquesPinheiro, Cooperativas de Crédito cit., p. 28.

5 Cf. M. Carvalhosa, ComentáriosaoCódigoCivil, vol. 13, Parte Especial. Do Direito de Empresa. Da SociedadePersonificada. Do Estabelecimento. DosInstitutosComplementares (Artigos 1.052 a 1.195), 2. ${ }^{a}$ ed., São Paulo, 2005, p. 394.

6 Cf. M. Carvalhosa, ComentáriosaoCódigoCivil cit., p. 394.

7 Cf. M. Carvalhosa, ComentáriosaoCódigoCivil cit., p. 394.

8 Cf. N. ReisJúnior, SociedadesCooperativas. LinhasGerais e AspectosSocietários, in F. Viana Rodrigues (coordenador), Direito de Empresa no Novo CódigoCivil, Rio de Janeiro, 2004, p. 372. 
natura come civile o commerciale, e avendo fatto appello a molte delle disposizioni relative alle società di tipo classico, il legislatore non ha osato considerarla, espressamente e chiaramente, un nuovo tipo di società. In ogni caso, venne meno, anche se in modo imperfetto, la subordinazione della società cooperativa alle forme delle altre società. ${ }^{9}$ Inoltre, questo documento legale ha ribadito la non obbligatorietà del capitale sociale, in particolare per le cooperative di tipo Raiffeisen, e ha portato una lunga lista di modalità di cooperative: di assicurazione, di alloggi, di scuole ecc.

Diverse iniziative legislative seguirono la norma del 1932, sempre, però, con l'intento di limitare la precedenti libertà d'azione delle cooperative. Prima revocato, poi ristabilito questo documento legale, si creò insicurezza nel settore. Tra le norme del periodo, si può citare il decreto-legge $\mathrm{n}^{\mathrm{o}} 581$, del $1^{\circ}$ agosto 1938 , che prevedeva la registrazione, la supervisione e l'assistenza alle società cooperative, e il decreto-legge $n^{\circ}$ 5.893, del 19 ottobre 1943, che si occupava dell'organizzazione, gestione e supervisione delle cooperative. ${ }^{10}$

Il successivo quadro giuridico rilevante per le cooperative è stato il decreto-legge $n^{0} 59$, del 21 novembre 1966, che ha definito la politica nazionale di cooperazione e ha creato il "ConselhoNacional do Cooperativismo", revocando il decreto no 22.239/1932. É stata inaugurata una fase di stretto controllo statale sul settore cooperativo brasiliano. É stata stabilita la obbligatorietà dell'autorizzazione del governo per il funzionamento delle cooperative e sono stati cancellati una parte dei benefici fiscali e del credito di cui beneficiavano, generando insicurezza e insoddisfazione nel settore. La ovvia conseguenza fu una profonda crisi del sistema cooperativo. ${ }^{11}$

Purtuttavia, bisogna riconoscere l'indubbio apporto positivo della norma del 1966, che conferì espressamente, per la prima volta, una forma societaria propria alla società cooperativa. ${ }^{12}$

In seguito, il decreto-legge $\mathrm{n}^{\circ} 59 / 1966$, è stato abrogato dalla legge $n^{0}$ 5.764, del 16 dicembre 1971, ancora in vigore, che definisce la "PolíticaNacional de Cooperativismo" e ha stabilito il quadro giuridico per la società cooperative, dando loro forma e caratteristiche proprie. In linea con quanto già intravisto con la legge precedente, la legge del 1973 sulla società cooperativa "representou, semdúvidaalguma, nítidaevolução no sentido de atribuirnaturezaprópria à cooperativa, inconfundívelcomasdemaisformasassociativas e societáriasatéentãoconhecidas no direitopátrio"13.

9 Cf. W. Bulgarelli, SociedadesComerciais. SociedadesCivis. SociedadesCooperativas.

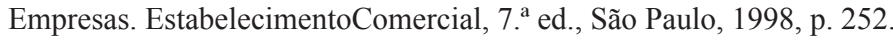

10 Cf. M. Carvalhosa, ComentáriosaoCódigoCivil cit., p. 394.

11 Cf. M. Carvalhosa, Comentáriosao CódigoCivil cit., p. 395.

12 Cf. W. Bulgarelli, SociedadesComerciais cit., p. 256.

13 Cf. A. Wald, Da Natureza e do Regime JurídicodasCooperativas e do SócioDemitidoouQue 
Si noti, per inciso, la definizione legale degli articoli $3^{\circ}$ e $4^{\circ}$ :

"Art. $3^{\circ}$ Celebram contrato de sociedade cooperativa aspessoasque reciprocamente se obrigam a contribuir combensouserviços para o exercício de umaatividadeeconômica, de proveitocomum, semobjetivo de lucro."

"Art. $4^{o}$ Ascooperativassãosociedades de pessoas, com forma e naturezajurídicapróprias, de naturezacivil, nãosujeitas a falência, constituídas paraprestar serviçosaosassociados, distinguindo-se dasdemaissociedadespelasseguintescaracterísticas (...)."

Si segnala, inoltre, come una delle principali innovazioni delle legge del 1971, il capitolo sul sistema operativo di cooperative, in cui si stabilisce, per esempio, che gli atti di cooperazione non costituiscono operazioni di mercato. ${ }^{14}$

D'altra parte, la legge $n^{0} 5.764 / 1971$, ha promosso, nei suoi 117 articoli, un'ampia regolamentazione del settore. Si è definita, per mezzo d'essa, la politica nazionale di cooperazione, assegnandosi al governo federale il coordinamento e l'incoraggiamento delle cooperative, attraverso il "ConselhoNacional de Cooperativismo". $\mathrm{Si}$ resero maggiormente dettagliati i meccanismi e gli organi di vigilanza e controllo del settore, mantenendo, al contempo, la previa autorizzazione per il funzionamento delle cooperative, come previsto dalla legislazione del 1966. Nonostante tale controllo del governo, la dottrina intravide, nella legge vigente, una "tendência à mitigação da intervençãoestatal no setor", dal momento che, "emdiversospontos da lei, percebe-se que a intençãofoiflexibilizargradativamenteasestruturas do cooperativismo", rappresentando tale flessibilità il punto di partenza di un periodo di rinnovamento del settore, che ha portato alla attuale fase della liberalizzazione delle cooperative, che ha avuto inizio con la Costituzione Federale del 1988. ${ }^{15}$

Infatti, la Costituzione del 1988 ha riconosciuto l'importanza delle cooperative e la loro indipendenza ${ }^{16}$, affermando che "a lei apoiará e estimulará o cooperativismo e outrasformas de associativismo" (art. 174, $\S 2^{\circ}$ ); disponendo un "adequadotratamentotributárioaoato cooperativo

se Retira da Sociedade, in RevistadosTribunais, vol. 711, São Paulo, janeiro de 1995, p. 64.

14 Cf. W. Bulgarelli, SociedadesComerciais cit., pp. 260-262.

15 Cf. M. Carvalhosa, ComentáriosaoCódigoCivil cit., pp. 395-396.

16 Cf. P.M. Pereira Corrêa da Fonseca \& R. Sztajn, CódigoCivilComentado, vol. XI, Direito de Empresa (Artigos 887 a 926 e 966 a 1.195), São Paulo, 2008, p. 596. 
praticadopelassociedadescooperativas" (art. 146, III, c); e inserendo una clausola di autonomia cooperativa, secondo la quale "a criação de associações e, na forma da lei, a de cooperativasindependem de autorização, sendovedada a interferênciaestatalemseufuncionamento.' (art. $\left.5^{\circ}, \mathrm{XVIII}\right)$. Essendo state le cooperative esentate, in sede costituzionale, della previa autorizzazione e dell'intervento dello Stato nel suo funzionamento, le disposizioni della legge 5.764/1971 che prevedono tale autorizzazione, chiaramente, non sono stateaccolte dalla Costituzione del $1988 .{ }^{17}$

Infine, la legge $\mathrm{n}^{\mathrm{o}} 10.406$, del 10 gennaio 2002, che ha istituito il nuovo Codice Civile brasiliano, ha dedicato, nell'ambito del Libro sulla Società, tra i tipi di società ammesse nel diritto brasiliano, una sezione per le società cooperative, senza rimuovere l'applicazione della legge speciale del 1971. Pertanto, le società cooperative sono attualmente disciplinate dalle disposizioni e dai principi costituzionali a loro inerenti, dalla legge $\mathrm{n}^{\circ}$ 5.764/1971, e del Codice Civile del $2002^{18}$ (e questo, ovviamente, senza ignorare le varie normative specifiche, sui vari tipi di cooperative). E certo che la legge speciale del 1971 e del Codice Civile devono coordinarsi, e ci possono essere alcunedifficoltà ermeneutiche nei casi di conflitto tra i diversi dispositivi. La dottrina è incline ad affermare la prevalenza del Codice Civile del 2002, essendo, da un punto di vista temporale, l'ultima legge sulla stessa materia, a partire dalla legge $n^{0} 5.764 / 1971$, revocando le disposizioni di quest'ultima ad esso contrarie. ${ }^{19}$

È necessario, ora, precisare che l'avvento del Codice Civile ha aperto una discussione sulla natura della società cooperativa. Se la legge $n^{\circ} 5.764 / 1971$ le conferiva, nel suo art. $4^{\circ}$, la condizione di tipo speciale di società, il Codice Civile la ha trattato, nel suo art. 982, par. singolo, come società semplice (società che non esercitano attività d'impresa). Sembra prevalere la posizione secondo cui le cooperative non sarebbero un tipo di società semplice, avendo una natura giuridica ibrida, tra le società civile e quelle che esercitano attività d'impresa, in tal modo la remissione citata del Codice Civile per le società semplici sarebbe solo un modo per la soluzione delle lacune nella legislazione speciale sulle cooperative (cioè, la legge $\mathrm{n}^{0} 5.764 / 1971$, fatte salve le disposizioni del Codice Civile del 2002). ${ }^{20}$

In sintesi, e al di là di una mero resoconto cronologico delle principali fonti normative, l'evoluzione storico-legislativa della

17 Cf. M. Carvalhosa, ComentáriosaoCódigoCivil cit., p. 396.

18 Cf. N. ReisJúnior, SociedadesCooperativas cit., pp. 372-373.

19 Cf. M. Carvalhosa, ComentáriosaoCódigoCivil cit.,p. 397; N. ReisJúnior, SociedadesCooperativas cit., p. 376; e H. MalheirosDuclercVerçosa, Curso de DireitoComercial, vol. 2, Teoria GeraldasSociedades. AsSociedadesemEspécie do CódigoCivil, São Paulo, 2006, pp. 544-545.

20 Cf. H. MalheirosDuclercVerçosa, Curso de DireitoComercial cit., pp. 545-546; e N. ReisJúnior, SociedadesCooperativas cit., p. 374. 
società cooperativa brasiliana, verificati i tratti distintivi che sono state descritti nei paragrafi precedenti, può essere organizzata secondo l'identificazione di alcuni periodi, che permettono di comprendere lo sviluppo globale del fenomeno, a partire dalle ripercussioni del proprio impatto sull'ambiente cooperativo ${ }^{21}$ : (i) periodo di impiantazione, che inizia con la prima legge organica nel 1907; (ii) periodo di consolidamento parziale, che inizia con la promulgazione del decreto del 1922; (iii) Periodo di centralismo statale, che è sancito dalla norma del 1966, e ha provocato una profonda crisi del sistema cooperativo brasiliano; (iv) Periodo del rinnovo delle strutture, con la legge del 1971, ancora in vigore, e, in ogni caso, nell'ambito di un sistema di coordinamento centrale dello Stato; e (v) Periodo di liberalizzazione, dopo la promulgazione della Costituzione del 1988.

\section{CARATteristiche Della SOCIETÀ COOPERATIVA BRASILIANA}

Da un lato, se l'elemento della cooperazione deve essere presente in tutte le società, dall'altro lato, la scelta della forma della società cooperativa per il tramite di agenti economici avrà rapporto con le sue caratteristiche specifiche, le quali, sotto molteplici aspetti, differiscono da quelle di altre figure aziendali.

La prima di queste caratteristiche, che comunque non è esclusivadella sola forma cooperativa, è la affectiosocietatis. Tuttavia, nel caso specifico della cooperativa, il fatto di essere una società di persone dà centralità all'elemento della affectiosocietatis. Nonostante alcune somiglianze di struttura organizzativa fra società cooperativa e la società anonima, la prima non perde la sua natura di società di persone, pur mantenendo una certa vicinanza con la natura della seconda (società di capitali). Poiché la cooperativa si basa sul legame personale tra i suoi membri, l'affectiosocietatis ha chiara natura intuitupersonae, cioè, è costituita sulla base delle caratteristiche e qualità individuali di ogni membro. ${ }^{22}$

$\mathrm{Ma}$, ancora una volta, al contrario di quanto avviene in altre strutture aziendali, nella cooperativa i cooperati hanno due funzioni, essendo soci e "clienti" o "fornitori", cioè, essendo produttori e consumatori di beni e servizi offerti dalla società cooperativa. ${ }^{23}$ Per quanto riguarda la integralizzazione del capitale, interessante osservare che i membri, secondo

21 Cf. W. Bulgarelli, A Sociedade Cooperativa e a Sua Disciplina Jurídica, Rio de Janeiro, 1988, pp. 63-76.

22 Cf. W. Bulgarelli, A Sociedade Cooperativa cit., pp. 54-55; M. Carvalhosa, ComentáriosaoCódigoCivil cit., p. 399; e F.C. Pontes de Miranda, Tratado de DireitoPrivado, vol. XLIX, Rio de Janeiro, 1965, p. 429.

23 Cf. A. Wald, Da Natureza e do Regime JurídicodasCooperativas cit., p. 63; N. ReisJúnior, SociedadesCooperativas cit., p. 373; e H. MalheirosDuclercVerçosa, Curso de DireitoComercial cit., p. 545. 
la situazione, sono obbligati o ad un contributo limitato o non sono tenuti ad alcun contributo (cooperative senza capitale sociale). ${ }^{24}$

Per questo motivo, si classifica la società cooperativa come quella società in cui i soci forniscono una "contribuição-patrimoniallimitadaouilimitada e contribuição-pessoal-máxima". Quest’ultimo elemento, inoltre, è essenziale per caratterizzare la cooperativa come una società di persone di eccellenza, dal momento che in nessun altro tipo di società la partecipazione dei soci non è cosí intensa,la qual cosa spiega anche il fatto che non sia classificata in dottrina come società mista, nonostante sia regolata, in concomitanza, dalle disposizioni delle società di capitali e da quelle delle società di persone..$^{25}$

Un'altra caratteristica importante di una società cooperativa è la variabilità o rinuncia del capitale. A differenza di altre aziende, in cui la indicazione di capitale sociale è elemento essenziale e indispensabile per la sua iscrizione nel registro delle imprese, questo non è elemento obbligatorio per la valida costituzione della cooperativa. Nel caso in cui ci sia l'indicazione del capitale, questo sarà variabile e potrà aumentare 0 diminuire a seconda della variazione del numero dei membri o delle azioni sottoscritte, senza modifiche di statuto o la manifestazione dell'assemblea. ${ }^{26}$

Per quanto riguarda il numero di soci, la legge richiede che esso sia illimitatorelativamente al numero massimo, ma dovrebbe rappresentare un numero minimo di soci sufficiente per ricoprire tutte le cariche di gestione cooperativa, in ogni caso mai essere inferiore a venti individui. Non si può negare che si tratta di una soluzione logica e efficace, perché con essa si garantisce la distribuzione fra i soci delle funzioni amministrative necessarie per svolgere l'attività d'impresa. Tuttavia, la dottrina non manca di sottolineare il pericolo intrinseco di tale disposizione di legge, non essendo quest'ultima in armonia con il principio costituzionale di adesione volontaria (art. $5^{\circ}, \mathrm{XX}$ ). Così, per rendere compatibile le disposizioni appena richiamate, è necessario che si interpreti la norma giuridica alla luce della norma costituzionale, affinché non si legga la disposizione di cui sopra come una mera costrizione o convinzione del socio della cooperativa ad associarsi od a rimanere nello status di associato, in virtù di qualsivoglialegame che si stabilisca con la cooperativa o funzione da effettuarsi nella cooperativa. D'altra parte, il principio appena esposto non deve essere interpretato in forma assoluta: ne consegue che è riconosciuto in capo alla cooperativa

24 Cf. W. Bulgarelli, A Sociedade Cooperativa cit., pp. 55-56; A. Wald, Da Natureza e do Regime JurídicodasCooperativas cit., p. 63.

25 Cf. W. Bulgarelli, A Sociedade Cooperativa cit., p. 56.

26 Cf. J. Batista Brito Pereira, Da Sociedade Cooperativa cit., p. 945; G. Mamede, DireitoEmpresarial Brasileiro, vol. 2, DireitoSocietário: SociedadesSimples e Empresárias, 4 a ed., São Paulo, 2010, pp. 589-590; N. ReisJúnior, SociedadesCooperativas cit., p. 376; e H. MalheirosDuclercVerçosa, Curso de DireitoComercial cit., p. 547. 
il diritto di rifiutare l'ingresso di persone che non siano in grado di sviluppare le attività della società o non accettino le responsabilità risultanti dell'adesione. ${ }^{27}$

Per quanto riguarda la quota dei soci, essa si caratterizza dalla non-trasferibilitàdelle azioni ai terzi. Cioè, a differenza di quanto accade con i membri delle altre società, $i$ soci della cooperativa non possono cedere proprie quote a persone che non siano già membri della cooperativa. In nessun caso è, difatti,permesso tale trasferimento, neanche in caso di trasferimento causa mortis ${ }^{28}$. Inoltre, la legge pone un limite, nel caso delle cooperative, al numero di quote del capitale sociale che ogni membro può sottoscrivere. La legge dispone che ciascuna quota non possaavere valore superiore al salario minimo più alto del paese e che il socio non possasottoscrivere più di un terzo delle quote (la qual cosatrova giustificazione nel fatto chenon è previsto fra gli scopi della società cooperativaquello di permetteread un socio di essere controllore di maggioranza delle quote, attraverso una partecipazione prevalente nel capitale rispetto ad altri soci) ${ }^{29}$.

Questa regola ha una connessione logica diretta con un'altra caratteristica delle società cooperative, ovvero la regola dell'unicità del voto (principio di gestione o di amministrazione democratica). In altre parole, nelle cooperative il socio ha diritto a un solo voto, indipendentemente dal numero di quote possedute. Per questo motivo, il quorum deliberativo nelle assemblee generali della società cooperativa non si basa sulla rappresentatività del capitale, ma sul numero dei soci. In tal modo, si garantisce anche che nell'assemblea generale si abbia l'effettiva partecipazione dei soci anche nelle cooperative in cui non c'è il capitale, dal momento che la fase deliberativa in tale organo non è correlata alla partecipazione economica dei soci. Inoltre, comeconseguenza di ciò, le decisioni in assemblea generale sono prese sempre a maggioranza dei voti, dal momento che ogni voto rappresenta la manifestazione della volontà di un solo socio, indipendentemente dal fatto che questi sia entrato nella cooperativa con un apporto economico al capitale sociale e quanto, da un punto di vista proporzionale, il valore dell'apporto rappresenti nella totalità del capitale sociale. ${ }^{30}$

27 Cf. J. Batista Brito Pereira, Da Sociedade Cooperativa cit., pp. 945-946; G. Mamede, DireitoEmpresarial Brasileiro cit., pp. 588-589; N. ReisJúnior, SociedadesCooperativas cit., p. 376; e H. MalheirosDuclercVerçosa, Curso de DireitoComercial cit., pp. 547-548.

28 Cf. J. Batista Brito Pereira, Da Sociedade Cooperativa cit., p. 947; e N. ReisJúnior, SociedadesCooperativas cit., p. 376.

29 Cf. J. Batista Brito Pereira, Da Sociedade Cooperativa cit., pp. 946-947; G. Mamede, DireitoEmpresarial Brasileiro cit., pp. 590-591; N. ReisJúnior, SociedadesCooperativas cit., p. 376; e H. MalheirosDuclercVerçosa, Curso de DireitoComercial cit., p. 548.

30 Cf. J. Batista Brito Pereira, Da Sociedade Cooperativa cit., pp. 947-948; G. Mamede, DireitoEmpresarial Brasileiro cit., pp. 591-592; N. ReisJúnior, SociedadesCooperativas cit., pp. 
Tuttavia, è importante notare che, per quanto riguarda la ripartizione della responsabilità tra i soci, non è rilevante, a priori, la partecipazione di questi nel capitale sociale, ma, piuttosto,rileva il livello di partecipazione alle operazioni dannose nei confronti di terzi, $\mathrm{o}$, in aggiunta, rileva la responsabilità illimitata, che, a secondadel caso, non impedisce che le quote-parte, quando esistano, possano servire da base per eventuali responsabilità. Dunque, l'ordinamento classifica le cooperative in limitate, in cui i soci rispondono solo per il valore del capitale sottoscritto e non ancora conferito, ed illimitate, dove il socio è responsabile personale, in solido ed illimitatamente per gli impegni di cooperazione, tuttavia, rispettandosi sempre, nel caso, il beneficio dell'ordine, in virtù del quale i soci possono essere responsabilisolo per obbligazioni inadempiute dalla società a favore di terzi, dopo che lo stesso debito sia stato richiesto in giudizio alla cooperativa. ${ }^{31}$

Per quanto riguarda la questione della distribuzione dei risultati tra i cooperati, si applica alle cooperative il cosiddetto principio del ritorno, secondo il quale i risultati sono distribuiti tra soci in proporzione al valore delle transazioni effettuate da loro nella normale attività della cooperativa. Inoltre, se c'è uncapitale sociale, lo statuto può prevedere che i soci ricevano una remunerazione fissa in ragione del capitale che hanno conferito o può anche prevedere l'assegnazioneun interesse fisso per lo stesso capitale versato. È importante, infine, ricordare che parte del surplus delle operazioni della cooperativa può essere assegnato alla costituzione di fondi, come il Fundo de Reserva e o Fundo de AssistênciaTécnica, Educacional e Social..$^{32}$

Un'altra caratteristica di una cooperativa è il suo carattere non imprenditoriale, che è strettamente connesso con la questione dello scopo lucrativo. La cooperativa, a differenza di altre società, è impegnata nel "viabilizar a atividadesocioeconômica de seusassociados", il che non implica la realizzazione di operazioni di qualunque attività economica specifica. La struttura organizzativa di un tale genussociale, dunque, è focalizzata sugli interessi dei soci, il che, non necessariamente, significa la ricerca del profitto. ${ }^{33}$

375-376; e H. MalheirosDuclercVerçosa, Curso de DireitoComercial cit., pp. 548-549.

31 Cf. G. Mamede, DireitoEmpresarial Brasileiro cit., p. 588; J. Batista Brito Pereira, Da Sociedade Cooperativa cit., p. 947; N. ReisJúnior, SociedadesCooperativas cit., pp. 381-382; e H. MalheirosDuclercVerçosa, Curso de DireitoComercial cit., pp. 547, 548 e 550.

32 Cf. H. MalheirosDuclercVerçosa, Curso de DireitoComercial cit., p. 549; J. Batista Brito Pereira, Da Sociedade Cooperativa cit., p. 948; e G. Mamede, DireitoEmpresarial Brasileiro cit., pp. 592-593.

33 Cf. J. Batista Brito Pereira, Da Sociedade Cooperativa cit., pp. 948-949; e W. Bulgarelli, Perspectivas da EmpresaPerante o DireitoEmpresarial - II (Conclusão: a Empresa Cooperativa), in Revista de DireitoMercantil, Industrial, Econômico e Financeiro, ano 11 (nova série), n. ${ }^{\circ}$, São Paulo, 1972, pp. 44-51. 
Questa definizione dell'oggetto della società cooperativa è un riflesso diretto del concetto di cooperativa elaborata nel XIX secolo e che rimane ancora oggi, ovverossia quello di solidarietà equidistante dall'individualismo capitalista e dalla soluzione comunista ${ }^{34}$. Questo risulta essere il cooperativismo antispeculativo. Oltre alle funzioni economiche essenziali, si ricercano anche obiettivi meta-economici, per esempio, di natura educativa e culturale, mettendo in primo piano non il capitale, ma la gente e il contributo personale di ciascun membro, con loscopo di miglioramento della situazione economica dei cooperati. Pertanto, si dice che la cooperativa ha carattere meramente strumentale, che si serve di un mezzo per raggiungere il suo scopo principale: la promozione dell'economia individuale dei soci. ${ }^{35}$

Questa caratteristica, a sua volta, è direttamente correlata alla natura giuridica di questo tipo di società. In realtà, la cooperativa è una persona giuridica di diritto privato, organizzata come una comunità di persone con finalità economica (e per questo è denominata società), ma non a scopo di lucro, come visto sopra. Il profitto è "remunerazione del capitale investito" e, dunque, in quanto non vi è necessariamente apporto di capitale da parte dei cooperati, non si deve parlare di profitto, che è remunerazione per gli investimenti. ${ }^{36}$

Le cooperative, oltre ad essere enti dotati di personalità giuridica (dalla registrazione del loro statuto nel registro delle imprese) e di scopo economico (per questo chiamate "società") senza scopo di lucro, sono società istituzionali, e non contrattuali, cioè, sono istituite e non contrattate. Tanto che le norme contenute negli statuti dell'organizzazione, l'attività degli organi e i diritti e i doveri dei soci, in relazione alla associazione, non hanno carattere contrattuale ma, piuttosto, normativo e istituzionale. ${ }^{37}$

Per quanto riguarda la struttura organizzativa e amministrativa della società cooperativa, l'assemblea generale è l'organo supremo, con poteri di decisione di tutte le questioni relative all'oggetto e alle prestazioni della società, dentro, tuttavia, i limiti statutari. Il quorum costitutivo è di almeno due terzi, in prima convocazione;della metà, in seconda convocazione (un'ora dopo la prima); e di dieci soci, in terza convocazione (un'ora dopo la seconda). La convocazione della assemblea compete al presidente, a qualsiasi organo di amministrazione

34 Cf. W. Franke, DireitodasSociedadesCooperativas (Direito Cooperativo), São Paulo, 1978, pp. 3-4; e W. Bulgarelli, Perspectivas da EmpresaPerante o DireitoEmpresarial cit., pp. 45-47. 35 Cf. J. Batista Brito Pereira, Da Sociedade Cooperativa cit., pp. 940-941 e 950-951; P.M. Pereira Corrêa da Fonseca \& R. Sztajn, CódigoCivilComentado cit., pp. 597-599; e W. Bulgarelli, Perspectivas da EmpresaPerante o DireitoEmpresarial cit., pp. 44-51.

36 Cf. G. Mamede, DireitoEmpresarial Brasileiro cit., pp. 586-587; A. Wald, Da Natureza e do Regime JurídicodasCooperativas cit., pp. 64-65.

37 Cf. G. Mamede, DireitoEmpresarial Brasileiro cit., pp. 586-587. 
(tra cui il consiglio fiscale) e agli stessi cooperati, a condizione che sianoalmeno il $25 \%$ del numero totale dei soci. ${ }^{38}$

Come accennato in precedenza, nell'assemblea generale la deliberazione è approvata dalla maggioranza, ogni socio dispone di un voto, indipendentemente dalla propria partecipazionenel capitale sociale. L'assemblea delibera sulle questioni avanzate dai soci presenti e la decisione è vincolante per tutti, anche i contrari e gli assenti, essendo, di regola, vietata la rappresentazione per procura. Se la deliberazione è viziata da errore, dolo, frode, simulazione o in violazione della legge o dello statuto, è concesso ai soci l'esercizio di un'azione per annullarla, soggetta al termine di prescrizione di quattro anni dalla deliberazione della precitata assemblea.

Sono previste due forme di assemblea generale, l'ordinaria e la straordinaria. La prima, secondo la legge, dovrebbe sempre essere convocata entro tre mesi dalla termine dell'anno fiscale e decide sui seguenti temi: la prestazione di conti degli organi di amministrazione accompagnata dal parere del "Conselho Fiscal"; l'assegnazione dei fondi rimanenti (dedotti da essi il valore delle parcelle destinate ai fondi obbligatori) o la ripartizione delle spese che hanno superato le ricorsi della società; l'elezione dei componenti degli organi di gestione, del "Conselho Fiscal" ecc.; la fissazione, se previsto, degli onorari, dei bonus e dello scrutinio della presenza dei membri del "Conselho de Administração" o della "Diretoria" e del "Conselho Fiscal"; altre questioni di interesse della società cooperativa. L'assemblea generale straordinaria, a sua volta, viene convocata quando è necessario e può decidere su tutte le questioni di interesse per la società, a condizione che sia elencato nel relativo ordine del giorno. Tuttavia, alcune questioni sono soltanto di competenza esclusiva dell'assemblea generale, vale a dire: modifica dello statuto; fusione, consolidamento o smembramento; cambio degli oggetto sociale; scioglimento volontario della società e nomina dei liquidatori; approvazione dei conti del liquidatore. L'adozione della decisione in una assemblea straordinaria richiede l'accordo di almeno due terzi dei soci presenti. ${ }^{39}$

L'amministrazione della cooperativa, a sua volta, è affidata alla "Diretoria" o al "Conselho de Administração" e, in conformità alle disposizioni dello statuto, i membri di tale organo sono solo cooperati eletti dall'assemblea generale. La durata del mandato di tali membri è determinata nello statuto, al massimo di quattro anni. Nello stesso statuto è prevista o meno la possibilità di rielezione del corpo amministrativo, tuttavia sempre nel rispetto della disposizione di legge che richiede la sostituzione di almeno un terzo dei membri. Lo statuto può anche prevedere la creazione di altri organi e consigli tecnici

38 Cf. G. Mamede, DireitoEmpresarial Brasileiro cit., p. 603.

39 Cf. G. Mamede, DireitoEmpresarial Brasileiro cit., p. 604. 
qualora si reputino utili o necessari alla gestione della società. Inoltre, è anche possibile che le organi dell'amministrazione si avvalgano di gerenti tecnici o commerciali che non sono membri della cooperativa. ${ }^{40}$

Gli amministratori, che siano essi eletti o gerenti tecnici o commerciali a contratto, agiscono come rappresentanti della società, in suo nome e con attenzione agli interessi propri e di quelli della collettività di cooperati. Di regola, non sono loro personalmente responsabili per le obbligazioni assunte in nome della cooperativa, a meno che il loro comportamentonon sia macchiato da dolo o colpa e causi pregiudizio alla società. In questo caso,è configurabile la responsabilità in solido degli amministratori. L'occultamento della natura della società può avere come conseguenza la responsabilità degli amministratori che hanno preso parte agli atti in questione. ${ }^{41}$

Al fine di controllarela condotta degli amministratori, la società cooperativa può avere un "Conselho Fiscal". Questo organo è composto da sei membri, tre effettivi e tre supplenti, tutti cooperati. L'elezione di questi membri si svolge ogni anno in occasione dell'assemblea generale, con la possibilità di rielezione solo di un terzo di loro. È vietato l'accumulo di posizioni in organi di gestionee di sorveglianza. ${ }^{42}$

Infine, si segnala che l'assemblea generale ha il potere di rimuovere i membri degli organi di amministrazione o di sorveglianza prima della fine del loro mandato. Se la rimozione di tali membri potrebbe causare qualche danno alle attività regolari degli organi di gestione della cooperativa, l'assemblea può nominare amministratori e consiglieri provvisori, fino all'entrata in carica dei nuovi membri permanenti, da eleggere entro trenta giorni. ${ }^{43}$

\section{NuMERI E DATI STATISTICI SULlE COOPERATIVE IN BRASILE}

L'importanza delle cooperative nello scenario economico brasiliano è già consolidata. Il numero di società cooperative, di soci e di funzionari e la fatturazione di essi possono essere presi in considerazione, nel presente elaborato, per avere una migliore visione del cooperativismo in Brasile.

I dati statistici sono stati raccolti dalla “OrganizaçãodasCooperativasBrasileiras" (OCB) ${ }^{44}$, del "ServiçoNacional

40 Cf. G. Mamede, DireitoEmpresarial Brasileiro cit., p. 605.

41 Cf. G. Mamede, DireitoEmpresarial Brasileiro cit., pp. 605-606.

42 Cf. G. Mamede, DireitoEmpresarial Brasileiro cit., p. 606.

43 Cf. G. Mamede, DireitoEmpresarial Brasileiro cit., p. 606.

44 Vedi, in particolare, il documento della OrganizaçãodasCooperativasBrasileiras, Panorama do Cooperativismo Brasileiro. Ano 2011, marzo del 2012, disponibile in $<$ http://www.ocb.org. br/gerenciador/ba/arquivos/panorama_do_cooperativismo_brasileiro__2011.pdf $>$.

Cf., anche, E. ScheidtNinaut\& M.A Matos, Panorama do Cooperativismo no Brasil, in 
de Aprendizagem do Cooperativismo" (Sescoop), e del "Ministério do Desenvolvimento, Indústria e Comércio Exterior", in materia di i) numero di cooperative, (ii) numero di soci, (iii) numero di funzionari, e (iv) partecipazione delle società cooperative in esportazioni brasiliane.

\subsection{I dati relativi al numero di società cooperative}

Secondo i dati forniti dalla $\mathrm{OCB}$, si può visualizzare il numero di cooperative esistenti in Brasile dal 2001 fino al 2011, in relazione al settore economico, alla regione e allo stato della federazione.

Nel 2011, si registra l'esistenza di 6.568 cooperative in Brasile. Dal 2001, vi è una certa variazione nel numero di società cooperative fino al 2008, quando si è arrivati ad un massimo di 7.682, e, quindi, in diminuzione fino al numero attuale (tabella 1). La riduzione del numero di cooperative non deve essere analizzata in modo negativo, poiché può essere letta come una ricerca di una maggiore competitività nel mercato: le cooperative si riuniscono per guadagnare scala e spazio, espandendo la propria attività, osservando, tuttavia, come si vedrà in articoli successivi, un aumento significativo della quantità di soci e funzionari, ovverossia, la forza di lavoro.

Tabella 1. Totale delle cooperative per anno:

\begin{tabular}{lccccccccccc}
\hline anno & 2001 & 2002 & 2003 & 2004 & 2005 & 2006 & 2007 & 2008 & 2009 & 2010 & 2011 \\
\hline totale di cooperative & 7.026 & 7.549 & 7.355 & 7.136 & 7.518 & 7.603 & 7.672 & 7.682 & 7.261 & 6.652 & 6.586 \\
\hline
\end{tabular}

I settori economici che più di altri sono caratterizzati dalla presenza delle società cooperative sono l'agricoltura (1.523), il trasporto (1.088) e il credito (1.047), conformemente a quanto mostrato nella tabella qui sotto:

\section{Grafico 1. Numero di cooperative per settore economico (2011)}

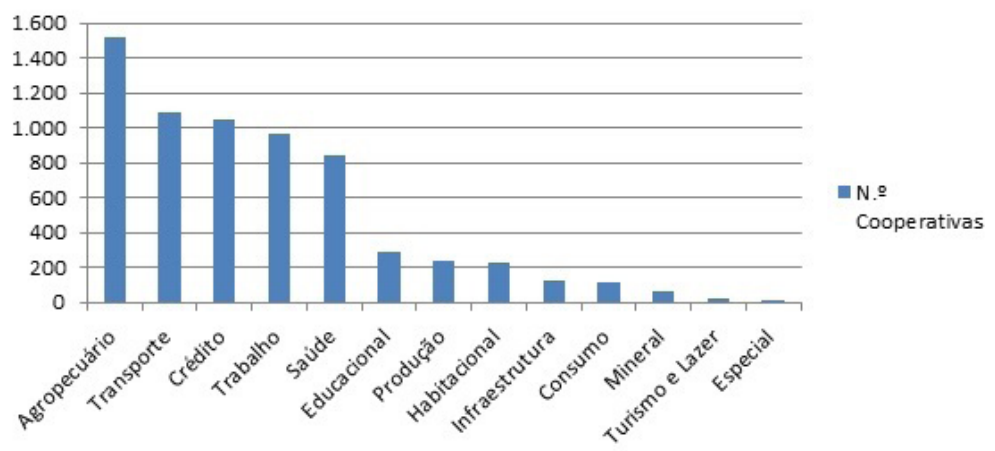

InformaçõesEconômicas, vol. 38, São Paulo, agosto de 2008, pp. 43-55, per un'analisi macroeconomica dei dati sul settore cooperativo per l'anno 2007. 
In fine, per quanto riguarda la distribuzione regionale, si nota che São Paulo è lo stato con il maggior numero di cooperative. Sono 932 le cooperative situate a São Paulo, seguite dalle 785 di MinasGerais, e dalle 783 di Bahia. Questi tre stati, insieme, hanno quasi il 30\% del numero di cooperative brasiliane, un dato interessante da considerare.

\section{Grafico 2. Numero di cooperative per stato della federazione (2011)}

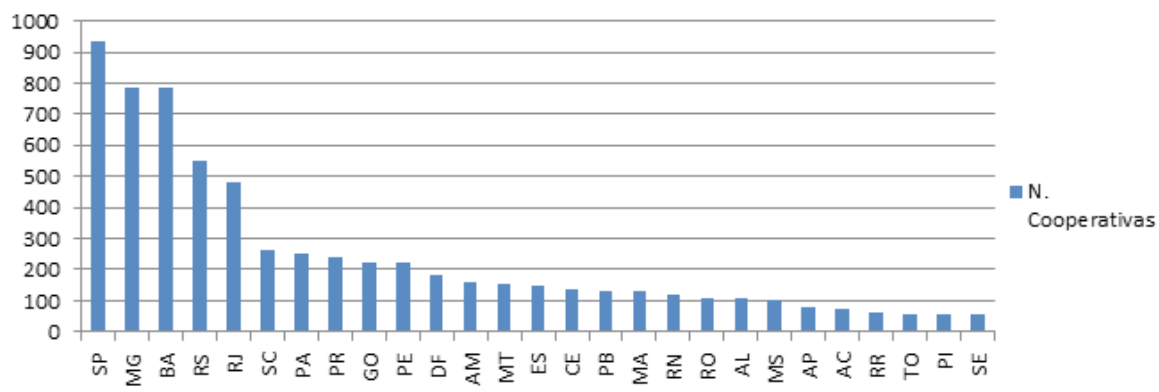

\subsection{I dati relativi al numero dei soci delle cooperative}

Il dato che più anima coloro che riconoscono la società cooperativa come un importante strumento di mercato è relativo al numero dei soci. Dal 2001, questo numero non ha mai smesso di crescere (grafico 1). Nel 2011 , il totale di soci delle cooperative vincolate alla OCB ha superato 10 milioni, con un incremento di $11 \%$ rispetto all'anno precedente. In 11 anni il numero è più che raddoppiato.

Tabella 2. Totale di soci per anno:

\begin{tabular}{cccc}
\hline anno & numero de cooperati & anno & numero de cooperati \\
\hline 2001 & 4.779 .000 & 2007 & 7.688 .000 \\
\hline 2002 & 5.259 .000 & 2008 & 7.888 .000 \\
\hline 2003 & 5.763 .000 & 2009 & 8.252 .000 \\
\hline 2004 & 6.160 .000 & 2010 & 9.017000 \\
\hline 2005 & 6.791 .000 & 2011 & 10.009 .000 \\
\hline 2006 & 7.393 .000 & & \\
\hline
\end{tabular}

Per quanto riguarda il settore economico, il settore di credito è quello nel quale ci sono più cooperati, essendoci più di quattro milioni di soci, seguito dal settore del consumo, con quasi tre milioni di cooperati, e dal settore agricolo, con un milione di cooperati. 
Grafico 3, Numero di soci per settore economico (2011)

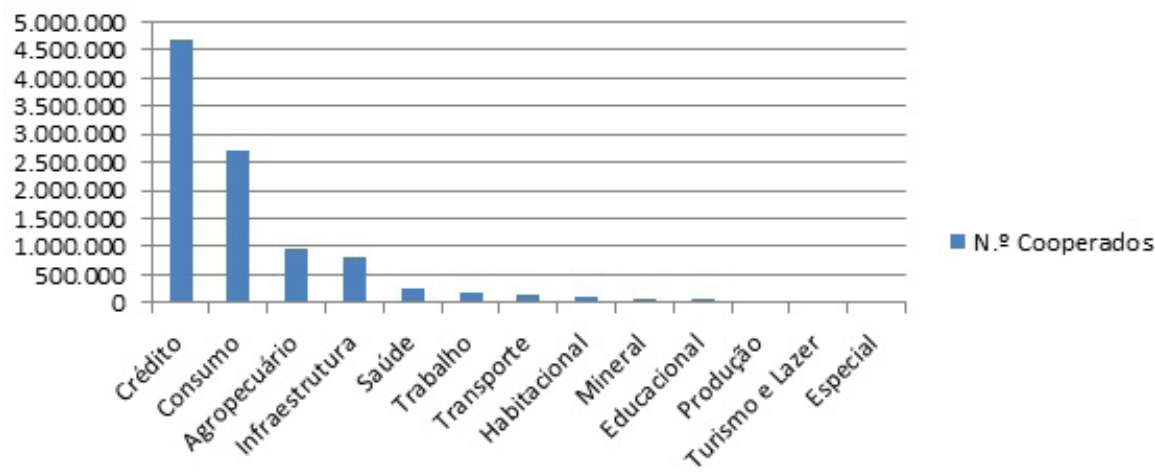

Per quanto riguarda la distribuzione regionale, São Paulo appare di nuovo come lo stato leader con riferimento al numero di soci, con tre milioni di membri, che rappresentano, di per sé, il 30\% del totale nazionale. Gli stati del Rio Grande do Sul e di Santa Catarina sono, rispettivamente, in $2^{\circ}$ e $3^{\circ}$ posizione, senza tuttavia riuscire a raggiungere, insieme, il totale di São Paulo. Lo stato di Santa Catarina, tuttavia, è un caso interessante perché ha una popolazione molto più piccola di altre unità federali che le sono dietro nel computo del numero di soci, e in Santa Catarina le cooperative sono responsabile pel 11\% del PIB.

\section{Grafico 4. Numero di soci per stato della federazione (2011)}

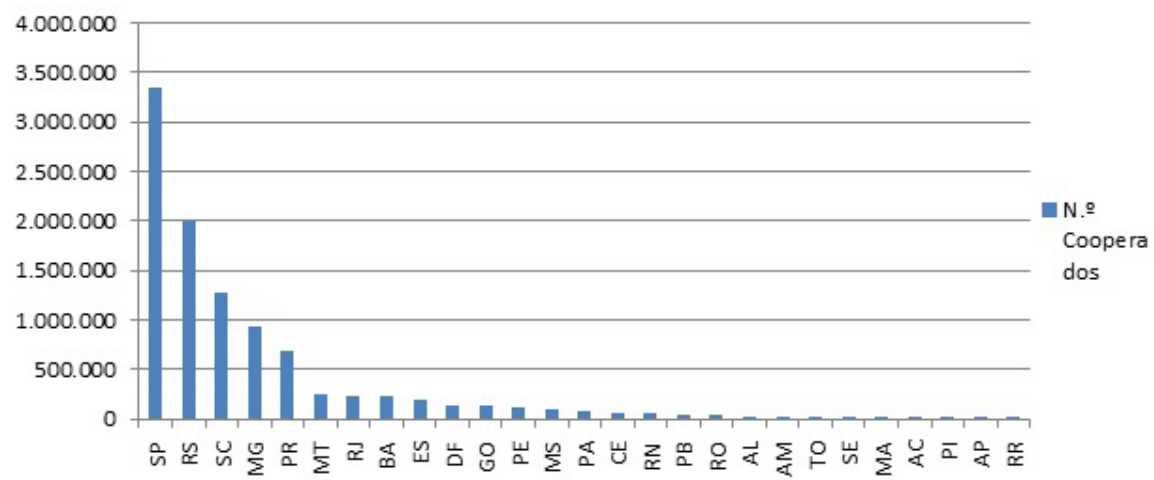

\subsection{I dati relativi al numero di funzionari di cooperative}

Anche il numero totale dei funzionari di cooperativecresce costantemente, considerando il periodo di analisi, tuttavia con minore intensità rispetto alla crescita del numero dei soci. Nel 2011, ci sono stati più di 296.000 funzionari di cooperative in Brasile. Ancora una volta il 
settore agricolo e di credito appaiono nelle primeposizioni: nel primo caso, sono più di 155.000 funzionari, e nel secondo, 34.000. Poi, in secondo piano vi è il settore sanitario, con 67.000 funzionari regolari, circa.

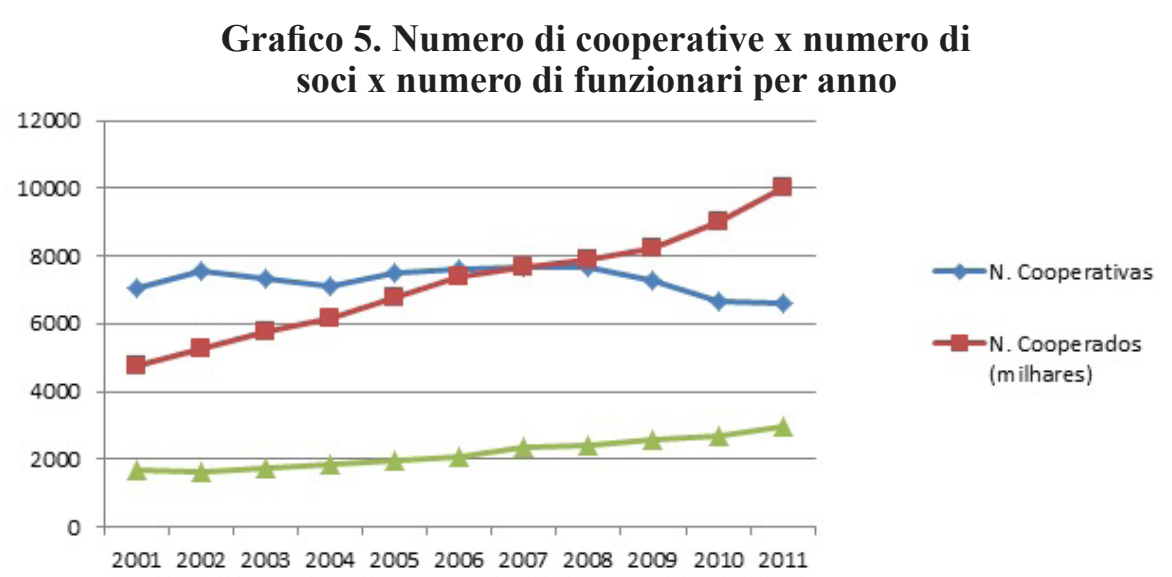

Per quanto riguarda la distribuzione regionale, stranamente, São Paulo, lo stato più ricco e popoloso del Brasile, appare solo al terzo posto, con 48.505 funzionari nelle proprie cooperative. Il leader è lo stato di Paraná, con 65.000 funzionari in cooperative, il secondo è lo stato del Rio Grande do Sul, con 48.755 funzionari.

\subsection{I dati sulle esportazioni delle cooperative}

I dati sulle esportazioni delle cooperative sono stati forniti dal "Ministério do Desenvolvimento, Indústria e ComércioExterior", analizzati dal 2006 al 2012. Le cifre sulla fatturazione sono stati forniti da OCB, avendo solo dati disponibili del 2002 al 2007.

Le esportazioni delle società cooperative hanno mostrato una crescita significativa in questo periodo, e gli unici periodi di diminuzione furono del 2011 al 2012, quando l'importo è passato da US\$ 256 miliardi a 242 miliardi, e dal 2008 al 2009. Di fronte al totale nazionale, tale valore è poco meno che $2,5 \%$ delle esportazioni brasiliane.

Per quanto riguarda il settore economico, più del $90 \%$ del totale riguarda prodotti agricoli, specie dello zucchero e della soia. Per quanto riguarda la distribuzione regionale, São Paulo è il leader di nuovo, con 34\% del totale delle esportazioni di cooperative, seguito da Paraná, con 29\%. 


\section{Grafico 6. Esportazioni delle cooperative per stato della federazione, in percentuale}

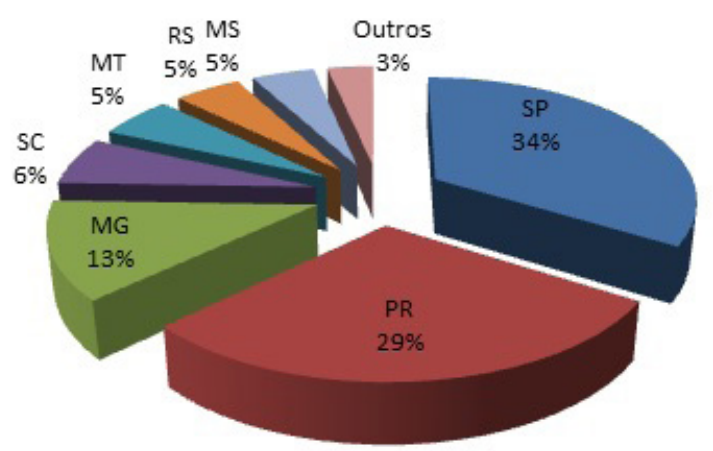

\section{RIFERIMENTI}

J. Batista Brito Pereira, Da Sociedade Cooperativa, in D. Franciulli Netto, G. Ferreira Mendes \& I. Gandra da Silva Martins Filho (coordenadores), O Novo Código Civil. Homenagem ao Professor Miguel Reale, 2. ${ }^{a}$ ed., São Paulo, 2006, pp. 937-963 [= in Revista do Tribunal Superior do Trabalho, vol. 69, n. ${ }^{\circ}$ 2, Brasília, julho-dezembro de 2003, pp. 32-54].

W. Bulgarelli, Perspectivas da Empresa Perante o Direito Empresarial II (Conclusão: a Empresa Cooperativa), in Revista de Direito Mercantil, Industrial, Econômico e Financeiro, ano 11 (nova série), n. ${ }^{\circ}$ 6, São Paulo, 1972, pp. 43-58.

W. Bulgarelli, Sociedades Comerciais. Sociedades Civis. Sociedades Cooperativas. Empresas. Estabelecimento Comercial, 7. ${ }^{\mathrm{a}}$ ed., São Paulo, 1998.

M. Carvalhosa, Comentários ao Código Civil, vol. 13, Parte Especial. Do Direito de Empresa. Da Sociedade Personificada. Do Estabelecimento. Dos Institutos Complementares (Artigos 1.052 a 1.195), 2. ${ }^{\mathrm{a}}$ ed., São Paulo, 2005.

W. Franke, Direito das Sociedades Cooperativas (Direito Cooperativo), São Paulo, 1978.

M.A. Henriques Pinheiro, Cooperativas de Crédito. História da Evolução Normativa no Brasil, 6. ${ }^{\mathrm{a}}$ ed., Brasília, 2008.

H. Malheiros Duclerc Verçosa, Curso de Direito Comercial, vol. 2, Teoria Geral das Sociedades. As Sociedades em Espécie do Código Civil, São Paulo, 2006. 
G. Mamede, Direito Empresarial Brasileiro, vol. 2, Direito Societário: Sociedades Simples e Empresárias, 4ª ed., São Paulo, 2010.

Ministério do Planejamento, Orçamento e Gestão, Instituto Brasileiro de Geografia e Estatística, Centro de Documentação e Disseminação de Informações, Brasil. 500 Anos de Povoamento, Rio de Janeiro, 2007.

Organização das Cooperativas Brasileiras, Panorama do Cooperativismo Brasileiro. Ano 2011, marzo del 2012.

P.M. Pereira Corrêa da Fonseca \& R. Sztajn, Código Civil Comentado, vol. XI, Direito de Empresa (Artigos 887 a 926 e 966 a 1.195), São Paulo, 2008.

F.C. Pontes de Miranda, Tratado de Direito Privado, vol. XLIX, Rio de Janeiro, 1965.

N. Reis Júnior, Sociedades Cooperativas. Linhas Gerais e Aspectos Societários, in F. Viana Rodrigues (coordenador), Direito de Empresa no Novo Código Civil, Rio de Janeiro, 2004, pp. 369-389.

E. ScheidtNinaut\& M.A Matos, Panorama do Cooperativismo no Brasil, in InformaçõesEconômicas, vol. 38, São Paulo, agosto de 2008, pp. 4355.

A. Wald, Da Natureza e do Regime Jurídico das Cooperativas e do Sócio Demitido ou Que se Retira da Sociedade, in Revista dos Tribunais, vol. 711, São Paulo, janeiro de 1995, pp. 63-72. 